\title{
Trauma et usage problématique de substances chez les jeunes : synthèse des travaux de recherche sur le programme Seeking Safety et projet pilote
}

Trauma and substance use among youth: A synthesis of research on the Seeking Safety program and pilot study Trauma y uso problemático de sustancias entre los jóvenes: síntesis de trabajos de investigación sobre el programa Seeking Safety y el proyecto piloto

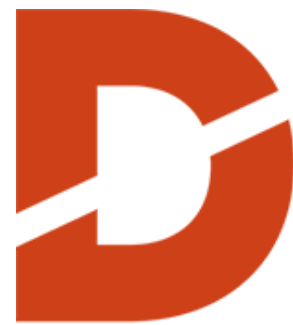

DROGUES, SANTÉ ET SOCIÉTÉ

\author{
Jean-Philippe É. Daoust, Lisa M. Najavits, Issack Biyong, Catherine Juéry et \\ Summer Krause
}

Volume 13, numéro 2, décembre 2014

Toxicomanie chez les jeunes : problèmes concomitants et pratiques à risque (1)

\section{URI : https://id.erudit.org/iderudit/1032275ar}

DOI : https://doi.org/10.7202/1032275ar

\section{Aller au sommaire du numéro}

\section{Éditeur(s)}

Drogues, santé et société

\section{ISSN}

1703-8847 (numérique)

\section{Découvrir la revue}

\section{Citer cet article}

Daoust, J.-P. É., Najavits, L. M., Biyong, I., Juéry, C. \& Krause, S. (2014). Trauma et usage problématique de substances chez les jeunes : synthèse des travaux de recherche sur le programme Seeking Safety et projet pilote. Drogues, santé et société, 13(2), 109-129. https://doi.org/10.7202/1032275ar

\section{Résumé de l'article}

Cet article porte sur le trouble concomitant état de stress post-traumatique (ÉSPT) et trouble lié à l'utilisation d'une substance (TUS) ; une réalité clinique fréquemment retrouvée chez les adultes où les traitements intégrés sont à privilégier comme modalité d'intervention. Parmi ceux-ci, le programme Seeking Safety (SS) est le plus validé empiriquement. Les preuves de son efficacité sont maintenant solides chez l'adulte et il est le seul traitement à surpasser le traitement contrôle pour l'ÉSPT et le TUS. Les travaux sur la version française sont aussi encourageants. Les jeunes, quant à eux, sont tout aussi à risque pour le type ÉSPT/TUS ; la prévalence étant estimée entre $11 \%$ et $47 \%$ (population générale et clinique). Bien que les impacts psychosociaux soient importants, peu de recherches portent sur les traitements intégrés pour cette clientèle. Deux études récentes mettent toutefois en évidence l'efficacité du programme SS pour les jeunes américains. Des ajustements sont d'ailleurs en cours pour adapter le programme à la réalité des adolescents et de leurs parents et pour en faciliter l'implantation. Des données préliminaires ont été récoltées auprès de jeunes canadiens-français (18 à 25 ans) qui ont bénéficié de séances d'entretien motivationnel combinées au programme $S S$ offertes en groupe $(n=6)$ ou en séances individuelles $(n=3)$. Les résultats suggèrent que la version francophone du programme $S S$ semble efficace en contexte de groupe, puisque des améliorations significatives ont été notées au niveau de la symptomatologie traumatique, de la consommation d'alcool et du fonctionnement selon l'axe 5 du DSM-IV-TR (grandes tailles d'effet). Aucune amélioration significative n'a cependant été notée pour la prestation de soins individuels et pour la consommation de drogues (DAST-20) en contexte de groupe ; bien que ces mesures semblent avoir évolué dans le sens d'un mieux-être. Cette étude suggère que la version française du programme $S S$ est prometteuse dans le traitement de l'ÉSPT-TUS pour une population clinique de jeunes canadien-français. Des études avec groupe contrôle et un plus grand nombre de participants devront toutefois être menées pour confirmer son efficacité. 


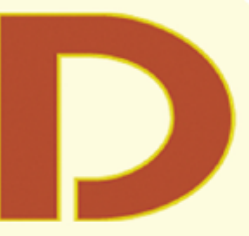

\section{Trauma et usage problématique de substances chez les jeunes : Synthèse des travaux de recherche sur le programme Seeking Safety et projet pilote}

Jean-Philippe É. Daoust, M.A.P., Ph.D., professeur adjoint et psychologue, Département de psychiatrie et Faculté d'éducation, Université d'Ottawa, Anciennement directeur clinique et de recherche de la Clinique Trauma et Psychiatrie Transculturelle

Lisa M. Najavits, Ph.D., professeure et psychologue, Department of psychiatry, Boston University School of Medicine

Issack Biyong, M.D., CTS, professeur adjoint, pédopsychiatre et psychiatre, Département de psychiatrie, Université d'Ottawa, Directeur médical et fondateur de la Clinique Trauma et Psychiatrie Transculturelle

Catherine Juéry, B.A.,Ps., M.Sc., ergothérapeute en santé mentale, Candidat au D.Psy en psychologie, Université du Québec en Outaouais

Summer Krause, MA, LPC, CADCIII, psychothérapeute en counseling, Western Psychological \& Counseling Services

\section{Correspondance:}

Dr Jean-Philippe É. Daoust

Programme de santé mentale

Hôpital Montfort

713 , chemin Montréal

Ottawa (Ontario)

K1K 0T2

Téléphone : 613 746-4621, poste 3926

Courriel : jpdaoust@uottawa.ca 


\section{Résumé}

Cet article porte sur le trouble concomitant état de stress post-traumatique (ÉSPT) et trouble lié à l'utilisation d'une substance (TUS); une réalité clinique fréquemment retrouvée chez les adultes où les traitements intégrés sont à privilégier comme modalité d'intervention. Parmi ceux-ci, le programme Seeking Safety (SS) est le plus validé empiriquement. Les preuves de son efficacité sont maintenant solides chez l'adulte et il est le seul traitement à surpasser le traitement contrôle pour l'ÉSPT et le TUS. Les travaux sur la version française sont aussi encourageants. Les jeunes, quant à eux, sont tout aussi à risque pour le type ÉSPT/TUS; la prévalence étant estimée entre $11 \%$ et $47 \%$ (population générale et clinique). Bien que les impacts psychosociaux soient importants, peu de recherches portent sur les traitements intégrés pour cette clientèle. Deux études récentes mettent toutefois en évidence l'efficacité du programme SS pour les jeunes américains. Des ajustements sont d'ailleurs en cours pour adapter le programme à la réalité des adolescents et de leurs parents et pour en faciliter l'implantation. Des données préliminaires ont été récoltées auprès de jeunes canadiens-français (18 à 25 ans) qui ont bénéficié de séances d'entretien motivationnel combinées au programme SS offertes en groupe $(n=6)$ ou en séances individuelles $(n=3)$. Les résultats suggèrent que la version francophone du programme SS semble efficace en contexte de groupe, puisque des améliorations significatives ont été notées au niveau de la symptomatologie traumatique, de la consommation d'alcool et du fonctionnement selon l'axe 5 du DSM-IV-TR (grandes tailles d'effet). Aucune amélioration significative n'a cependant été notée pour la prestation de soins individuels et pour la consommation de drogues (DAST-20) en contexte de groupe; bien que ces mesures semblent avoir évolué dans le sens d'un mieux-être. Cette étude suggère que la version française du programme SS est prometteuse dans le traitement de l'ÉSPT-TUS pour une population clinique de jeunes canadien-français. Des études avec groupe contrôle et un plus grand nombre de participants devront toutefois être menées pour confirmer son efficacité.

Mots-clés : état de stress post-traumatique, troubles liés aux substances, comorbidité, traitement intégré, jeune, français 


\title{
Trauma and substance use among youth: A synthesis of research on the Seeking Safety program and pilot study
}

\begin{abstract}
This article focuses on post-traumatic stress disorder (PTSD) and substance use disorder (SUD) as a concurrent disorder; a clinical reality frequently found in adults where integrated treatments are recommended as a method of intervention. Among them, the Seeking Safety program (SS) is the most empirically validated. Evidence of its efficacy is now established in an adult population and it is the only treatment that exceeds the control treatment for PTSD and SUD. The work on the French version is also encouraging. Youth, meanwhile, is equally at risk for this type of disorder; the prevalence is estimated between $11 \%$ and $47 \%$ (community and clinical population). Although psychosocial impacts are important, there is little research on integrated treatment for this population. However, two recent studies demonstrate the efficacy of the SS program for American youth. Adjustments are underway to adapt the program to the reality of adolescents and their parents and facilitate its implementation. Preliminary data were collected from French Canadian youth (18-25 years) who received motivational interviewing sessions combined with SS offered in group $(n=6)$ or in individual sessions $(n=3)$. The results show that the French version of the SS program appears to be effective in a group context as significant improvements were noted in the PCL-S, the MAST-22 and also according to the axis 5 of the DSM-IV-TR (large effect sizes). However, no significant improvement was noted for individual care and the DAST-20 in group context; although these measures appear to have evolved in the direction of wellness. This study suggests that the French version of the SS program is promising in the treatment of PTSD-SUD for French-Canadian youth in a clinical context.
\end{abstract}

Keywords: post-traumatic stress disorder, substance use disorders, comorbidity, integrated treatment, youth, French 


\section{Trauma y uso problemático de sustancias entre los jóvenes: síntesis de trabajos de investigación sobre el programa Seeking Safety y el proyecto piloto}

\section{Resumen}

Este artículo se refiere al transtorno de estrés postraumático (TEPT) y al problema ligado a la utilización de una sustancia como problema concomitante; una realidad clínica que se encuentra frecuentemente en los adultos, con los que se privilegia un tratamiento integrado como modalidad de intervención. Entre estos, el programa Seeking Safety (SS) es el que ha recibido más validación empírica. Las pruebas de su eficacia con los adultos son ahora sólidas y el programa constituye el único tratamiento que supera el tratamiento control del transtorno de estrés posttraumatico/TUS y el TUS. Los trabajos sobre la versión francesa son asimismo alentadores. Lo jóvenes, por su parte, están también sujetos a riesgo de presentar TEPT/TUS, estimándose la prevalencia entre el 11 y el $47 \%$ (población general y clínica). Si bien los impactos psicosociales son importantes, pocas son las investigaciones que se realizaron sobre los tratamientos integrados para esta clientela. Dos estudios recientes ponen sin embargo en evidencia la eficacia del programa SS para los jóvenes estadounidenses. Se están realizando ajustes para adaptar el programa a la realidad de los adolescentes y de sus padres y para facilitar su implantación. Se han recabado datos preliminares entre los jóvenes franco-canadienses (18 a 25 años) que han obtenido sesiones de entrevista motivacional combinadas con el programa SS que se ofrece en grupo $(n=6)$ o en sesiones individuales $(n=3)$. Los resultados sugieren que la versión francófona del programa SS parece eficaz en contexto de grupo, ya que se notaron mejoras significativas en lo que se refiere a la sintomatología traumática, el consumo de alcohol y el funcionamiento según el eje 5 del DSM-IV-TR (grandes medidas de efecto). Sin embargo, no se ha notado ninguna medida significativa con respecto a la prestación de atención individual ni al consumo de drogas (DAST 20) en contexto de grupo: aunque estas medidas parecen haber evolucionado hacia una mejora del bienestar. Este estudio sugiere que la versión francesa del programa SS es prometedora para el tratamiento del TEPT-TUS en el caso de una población clínica de jóvenes franco-canadienses. Deben realizarse sin embargo estudios con grupos de control y una mayor cantidad de participantes para confirmar su eficacia.

Palabras clave: transtorno de estrés postraumático, trastornos ligados al consumo de sustancias, comorbilidad, tratamiento integrado, joven, francés 


\section{Introduction}

Au fil des années, les données probantes ont fait état d'une grande prévalence de troubles concomitants dans la population clinique. Selon le DSM-5 (APA, 2013), un trouble concomitant se définit par la présence simultanée d'un trouble de santé mentale et d'un trouble lié à l'utilisation d'une substance (TUS).

À titre illustratif, une importante étude réalisée aux États-Unis (Reiger, Farmer \& Rae, 1990) et rapportée par Skinner, O'Grady, Bartha et Parker (2004) précise que 30\% des personnes qui sont diagnostiquées avec un trouble de santé mentale présentent aussi un TUS. Par ailleurs, $37 \%$ des individus aux prises avec un trouble lié à l'abus d'alcool et $53 \%$ des gens ayant un trouble lié à l'abus de drogues présenteraient un problème de santé mentale au cours de leur vie (Skinner et al., 2004); ce qui est respectivement deux fois et quatre fois supérieur aux individus ne présentant pas un TUS.

Dans le cadre de cette étude pilote, l'accent a été mis sur une réalité clinique fréquemment rencontrée, à savoir un état de stress post-traumatique (ÉSPT) combiné à un TUS. En fait, parmi les individus adultes présentant un ÉSPT, une comorbidité de TUS est présente dans $60 \%$ à $80 \%$ des cas (Donovan, Padin-Rivera \& Kowaliw, 2001). De plus, jusqu'à $80 \%$ des femmes cherchant un traitement pour un TUS rapporteraient avoir aussi subi des agressions sexuelles et/ou physiques (Brady, Killeen, Saladin, Dansky \& Becker, 1994; Dansky, Saladin, Brady, Kilpatrick \& Resnick, 1995; Fullilove, et al., 1993; Hien \& Scheier, 1996; Miller, Downs \& Testa, 1993). De même, le taux de TUS à vie est presque le double pour les gens aux prises avec un ÉSPT (43\%) que pour les individus qui ne présentent pas de symptômes d'ÉSPT (22\%) (Breslau, Davis, Andreski, Peterson \& Schultz, 1997 ; Cottler, Compton, Mager, Spitznagel \& Janca, 1992; Kessler, Sonnega, Bromet, Hughes \& Nelson, 1995; Kulka et al., 1990).

\section{Pertinence de la problématique}

La comorbidité ÉSPT-TUS soulève un intérêt croissant (Najavits \& Hien, 2013; Simoneau \& Guay, 2008; Skinner et al., 2004). En lien avec la prestation de soins sensibles (trauma informed care), la population générale a notamment été sensibilisée aux troubles traumatiques et à la présence de comorbidité. Stewart et Conrod (2008) rapportent toutefois que l'ÉSPT est encore souvent sousdiagnostiqué chez les individus qui recherchent de l'aide pour leur TUS.

Afin d'offrir un soutien thérapeutique aux individus dans le besoin, des programmes d'intervention se sont développés et ont été empiriquement étudiés. Quatre grandes classes de traitements peuvent être distinguées. La première classe de traitement se limite au traitement d'une des deux problématiques cliniques (ÉSPT ou TUS) sans tenir compte de l'autre (Hien, 2009). La deuxième classe de traitements porte sur le traitement en séquence des deux problématiques (souvent le TUS en premier) et ne s'intéresse qu'à une seule à la fois. Les partisans de ce type de traitement en séquence rapportent que le traitement de l'ÉSPT doit seulement débuter après une période d'abstinence prolongée (Ouimette, Moos et Brown, 2003 dans Riggs \& Foa, 2008). Toujours dans cette perspective, le traitement préalable du TUS permettrait aux patients d'acquérir des ressources (stratégies d'adaptations, soutien social, etc.) qui seraient bénéfiques au traitement subséquent de I'ÉSPT. Cette philosophie semble logique en théorie, mais il y aurait souvent des rechutes associées à ce type de programme, car les participants tenteraient de gérer leur détresse émotionnelle associée à l'ÉSPT non traité avec une utilisation de substances (Riggs \& Foa, 2008). Une troisième classe de thérapies propose un traitement en parallèle de l'ÉSPT et du TUS. Ce type de thérapies 
vise le traitement des deux troubles en même temps, et ce, dans des institutions indépendantes. Malheureusement, des difficultés de communication semblent être source d'inconsistance entre les deux traitements. Une dernière classe de traitement, d'apparition plus récente, est porteuse d'espoir, puisqu'elle aborde le traitement simultané et par une même équipe des difficultés ÉSPTTUS. Ces traitements, dits "intégrés" sont bien accueillis par les participants et même privilégiés par ces derniers (Brown, Stout \& Gannon-Rowley, 1998 dans Riggs \& Foa, 2008). De plus, des données probantes faisant preuve de leur efficacité sont maintenant établies (Riggs \& Foa, 2008) et reconnues dans les divers milieux cliniques.

Dans le contexte des traitements intégrés, quelques programmes ont été élaborés. De par sa disponibilité en français, la présente étude porte sur le programme "Seeking safety" (SS; Najavits, 2002a). II prend appui sur l'approche cognitive behaviorale et a pour but d'enseigner aux patients des stratégies d'adaptation sécuritaires (Riggs \& Foa, 2008). II ne repose pas sur les techniques d'exposition répétée, puisqu'il constitue une première phase de traitement (Najavits, 2003; Zlotnick, Najavits, Rohsenow \& Johnson, 2003). Dans une deuxième phase (Creating Change; Najavits, en préparation), l'exploration approfondie des traumatismes sera mise de l'avant.

Disponible sous forme de manuel de traitement, le programme SS est le premier traitement ÉSPTTUS pour lequel des résultats positifs ont été rapportés (Hien et al., 2004; Najavits, 2003). II est aujourd'hui empiriquement validé aux États-Unis pour les anglophones et plusieurs études ont été réalisées sur des populations variées : des femmes incarcérées (Lynch, Heath, Matthews \& Cepeda, 2012 ; Zlotnick, Johnson \& Najavits, 2009; Zlotnick et al., 2003), des femmes provenant de milieux défavorisés (Cohen \& Hien, 2006; Hien et al., 2004), des hommes et femmes vétérant(e) s de l'armée (Boden et al., 2011; Cook, Walser, Kane, Ruzek \& Woody, 2006; Desai, Harpaz-Rotem, Najavits \& Rosenheck, 2009; Desai, Harpaz-Rotem, Najavits \& Rosenheck, 2008; Norman, Wilkins, Tapert, Lang \& Najavits, 2010; Weaver, Trafton, Walser \& Kimerling, 2007; Weller, 2005) et des patients en clinique ambulatoires (Najavits, Schmitz, Gotthardt \& Weiss, 2005; Najavits, Weiss, Shaw \& Muenz, 1998).

En résumé, à la suite d'un récent relevé de littérature (Najavits \& Hien, 2013), il apparaît que les traitements intégrés ÉSPT-TUS donnent des résultats positifs dans plusieurs domaines de fonctionnement psychosocial. Cela dit, la plupart des programmes ont plus d'impact positif sur le plan du rétablissement de l'ÉSPT; ce qui suggère que le TUS est plus difficile à traiter. En ce qui concerne le programme SS, les preuves de son efficacité sont maintenant solides. II s'agit, en fait, du traitement intégré qui est le plus validé empiriquement et il est le seul qui surpasse le traitement contrôle pour l'ÉSPT et le TUS chez les adultes. II est à noter ici que le recours à tous les 25 modules du programme est à privilégier puisque l'utilisation de seulement certains modules du programme fait état de résultats mitigés (Najavits \& Hien, 2013). II importe également de mentionner que le programme SS s'est avéré bénéfique auprès d'échantillons cliniques complexes généralement exclus des études "étalon-or" portant sur le traitement unique de l'ÉSPT. Le traitement intégré EST/TUS requiert cependant plus de temps que le traitement unique de I'ÉSPT ou du TUS.

Bien que le programme soit disponible en français depuis 2008 (À la recherche de la sécurité), une seule étude publiée à ce jour semble être venue en soutenir les fondements empiriques pour une population francophone adulte (Daoust, Renaud, Bruyère, Juéry \& Najavits, 2014). 


\section{ÉSPT et TUS chez les jeunes}

Bien que peu étudiés empiriquement, les jeunes sont tout aussi à risque de présenter un trouble concomitant ÉSPT-TUS. En fait, il a été estimé que la prévalence de ce trouble se situe entre $11 \%$ (générale) et $47 \%$ (clinique) chez la population adolescente (Giaconia et al., 2000; Giaconia et al., 2002). Les adolescentes seraient particulièrement à risque, puisqu'elles présenteraient un plus haut taux d'ÉSPT que les adolescents (Kessler et al., 1995) et que leur taux d'utilisation de substances serait comparable à celui des adolescents (Najavits, 2002b). En termes cliniques, les individus aux prises avec un trouble concomitant feraient état d'un fonctionnement psychosocial plus détérioré que ceux présentant un seul diagnostic, et ce, tant chez les adolescents que les adultes (Giaconia et al., 2002; Ouimette \& Brown, 2002).

Les données issues du National Survey of Adolescents des États-Unis et d'autres études (voir Kilpatrick \& Saunders, 1997) indiquent qu'un enfant/adolescent sur quatre a fait l'expérience d'au moins un événement potentiellement traumatique avant l'âge de 16 ans et plus et que $13 \%$ des 17 ans et plus ont été aux prises avec un ÉSPT à un moment donné dans leur vie. Beaucoup de ces jeunes ont également accès à des substances psychoactives qui peuvent à la fois atténuer les effets du stress vécu et les mettre à risque de subir des traumatismes. II est en fait estimé que $29 \%$ des adolescents ont consommé des drogues illicites au moment où ils terminent la huitième année (secondaire 2) et que $41 \%$ d'entre eux ont alors consommé de l'alcool (Kilpatrick \& Saunders, 1997).

Plus près de chez nous, selon le Sondage sur la consommation de drogues et la santé des élèves de I'Ontario (Paglia-Boak, Adlaf, Hamilton, Beitchman, Wolfe \& Mann, 2012; Paglia-Boak, Adlaf \& Mann, 2011), 54,9\% des jeunes de la $7^{\mathrm{e}}$ à la $12^{\mathrm{e}}$ année ont consommé de l'alcool au cours de la dernière année et $37,4 \%$ se sont adonnés à une utilisation de substances illégales; y compris l'usage non médical (UNM) de médicaments d'ordonnance.

Le lien entre les abus physiques et sexuels de l'enfance, I'ÉSPT et le TUS subséquent est documenté depuis un certain temps dans la littérature portant sur les adolescents et les jeunes adultes (Briere \& Zaidi, 1989; Cohen, Spirito \& Sterling, 1996; Herman, Russell \& Trocki, 1986; Miller, Downs, Gondoli \& Keil, 1987). Malheureusement, et bien que les impacts psychosociaux soient importants, peu de recherches portent sur les traitements propres à cette clientèle.

Une revue de la littérature effectuée par Cohen, Mannarino, Zhitova et Capone (2003) rapporte que le seul traitement intégré de l'ÉSPT et du TUS chez les adolescents est le programme SS. Deux études relativement récentes mettent d'ailleurs en évidence le potentiel d'efficacité du programme SS pour les adolescents et les jeunes (18-25 ans) américains.

Une première étude (Najavits, Gallop \& Weiss, 2006) au devis clinique randomisé (SS vs traitement comme à l'habitude) a été menée auprès d'adolescentes $(\mathrm{N}=33)$, et ce, dans un format individuel. L'adhésion au manuel de traitement SS a été respectée le plus possible tout en aménageant les modifications suivantes : (1) communication verbale du contenu des modules si l'adolescente résistait à la lecture du matériel, (2) discussion en termes plus "dépersonnalisés" afin de faire évoquer des sentiments profonds de façon moins menaçante (par ex. : Que se passerait-il si cela arrivait à une bonne amie?), (3) discussion des détails de l'événement traumatique si l'adolescente en faisait la demande, (4) offre jusqu'à deux séances portant sur des sujets non couverts par le manuel de traitement et (5) offre de brèves mises à jour aux parents si l'adolescente acceptait. 
La durée du traitement était de 3 mois (période comparable pour le traitement comme à l'habitude : AA, pharmacothérapie, psychothérapie individuelle ou de groupe ou combinaison). Les 25 thèmes du programme ont été couverts par l'entremise de session de 50 minutes chacune. Les participantes des deux groupes (âge moyen de 16,1 ans) ont reçu un paiement symbolique pour la période d'évaluation (prétraitement, post-traitement et rencontre de suivi) alors que le programme SS était gratuitement offert.

Le programme SS s'est révélé supérieur au traitement comme à l'habitude (améliorations significatives : utilisation de substances et problèmes associés, certains symptômes traumatiques, cognitions en lien avec ÉSPT et TUS et quelques sphères pathologiques non directement touchées par le programme- par ex.: anorexie et somatisation). Les tailles d'effet observées variaient généralement de moyennes à grandes. Certains gains s'étaient aussi maintenus au moment du suivi à 3 mois. Bien que fort prometteur pour les jeunes, le besoin d'études empiriques complémentaires se fait sentir; de même que des adaptations cliniques au manuel de traitement.

Il est à noter qu'au moment de rédiger cet article, des ajustements au programme SS étaient en cours (Najavits \& Krause, en développement). En fait le programme SS est utilisé avec les jeunes depuis de nombreuses années en clinique et l'objectif de la version adaptée et de le rendre encore plus attrayant pour ce groupe d'âge. Ainsi, des activités à caractère ludique et créatif seront intégrées au contenu du manuel de base. Par exemple, en lien avec le thème de la sécurité, les adolescents pourront réaliser un collage d'images et de mots qui représentent la " sécurité " pour eux. Pour le module en lien avec l'intégration du moi divisé, les adolescents pourront créer une sorte de casse-tête où chaque pièce représentera une partie d'eux-mêmes. Pour le module sur l'ÉSPT, un jeu-questionnaire sera disponible afin de faciliter la psychoéducation. De façon générale, la documentation sera aussi simplifiée et incorporera des graphiques, des tableaux, des auto-évaluations et des exercices interactifs qui faciliteront l'apprentissage. De plus, chaque module inclura une "carte synthèse" décrivant les concepts clés que les adolescents pourront conserver avec eux pour une utilisation en dehors des séances.

Puisque les parents ou les autres dispensateurs de soins jouent souvent un rôle important dans le processus de rétablissement des adolescents (et ont souvent aussi leur propre histoire de traumatisme et de TUS), une section de chaque module sera consacrée à la façon dont ils peuvent soutenir leur adolescent; tout en identifiant la manière dont le sujet peut se rapporter à leur propre vie.

Des séances de groupe avec les parents pourront aussi être proposées. À ce propos, Cohen et ses collaborateurs (2003) proposent d'inclure davantage les parents dans les modalités de traitement et se réfèrent en ce sens aux recommandations émises dans le traitement intégré de la dépression et du TUS chez les adolescents (Riggs \& Davies, 2002). II apparait en fait que l'ajout d'une composante parentale présente des bénéfices significatifs dans le traitement de l'abus sexuel (Deblinger \& Heflin, 1996; Deblinger, Lippman \& Steer, 1996) et du TUS (Liddle \& Hogue, 2001).

En plus de la composante parentale, Riggs et Davies (2002) émettent aussi des recommandations en lien avec : (1) l'évaluation psychologique exhaustive, (2) l'utilisation de techniques d'entretien motivationnel pour faciliter la création d'une alliance thérapeutique solide et favoriser la réduction des méfaits, (3) le recours à des traitements validés pour le TUS, (4) l'utilisation d'une pharmacothérapie appropriée (avec monitorage de la toxicologie urinaire, évaluation de conformité à la prescription et suivi de la réponse symptomatique), (5) le recours à des traitements empiriquement validés (individuel ou familial), (6) la référence simultanée à un programme en douze étapes pour certains adolescents, (7) l'interruption et la référence à un traitement plus intensif s'il n'y a pas 
d'amélioration après les deux premiers mois de suivi et finalement, (8) I'utilisation de techniques de prévention de la rechute.

Toujours en lien avec la validation du programme SS pour les jeunes, une deuxième étude (Hamilton, 2006; Najavits, Schmitz, Johnson, Smith, North, Hamilton, Walser, Reeder, Norman, \& Wilkins, 2009) s'est intéressée aux jeunes hommes afro-américains (18-25 ans). Ces jeunes ( $N=76)$ recevaient des services dans le cadre d'un traitement résidentiel et étaient impliqués dans le système judiciaire pénal. Ils présentaient tous des problèmes sérieux au niveau de la santé mentale (17\% étaient aux prises avec un ÉSPT et $76 \%$ avaient été "victimisés" au cours de leur vie) et du TUS ( $80 \%$ rencontraient les critères pour un abus/dépendance à une substance). Le suivi s'est échelonné sur une période de six mois où tous les participants ont couvert l'ensemble des modules SS assignés.

Les résultats auto-rapportés ont fait état d'une diminution significative dans l'utilisation de substances (prétraitement : 25,3 jours de consommation en moyenne par mois; post-traitement : $100 \%$ d'abstinence; suivi à 12 mois $(\mathrm{N}=55)$ : 2 jours de consommation en moyenne par mois). Des améliorations significatives ont aussi été notées au niveau de l'échelle d'anxiété du SCL-90-R, de toutes les échelles du Cognitive Distorsions Scale (Briere, 2000), notamment sur les éléments suivants : tendance à l'autocritique, tendance à se blâmer, sentiment d'impuissance, désespoir et préoccupation avec le danger et de sept échelles du Trauma Symptom Inventory (Briere, 1995) : activation anxieuse, dépression, colère/irritabilité, expériences de reviviscence, comportements sexuels dysfonctionnels, autoréférence dysfonctionnelle et comportement de réduction de tension). Aucune donnée n'était toutefois disponible pour ces échelles au moment du suivi à 12 mois.

Les principales conclusions de cette étude sont à l'effet que le programme SS semble efficace pour les jeunes hommes criminels âgés de 18-25 ans dans leur compréhension des enjeux inhérents au trauma et des liens avec le TUS. II semble, par ailleurs, que le programme SS est en mesure d'offrir un environnement sécuritaire et propice au traitement résidentiel; bien qu'il soit nécessaire de mieux comprendre et de mieux planifier l'après-traitement résidentiel.

En résumé, le programme SS est non seulement disponible pour venir en aide aux jeunes aux prises avec un ÉSPT-TUS, mais il existe certaines preuves de son efficacité dans une application anglophone.

La dernière partie de cet article est consacrée à une évaluation très préliminaire d'un programme de traitement disponible pour les Franco-ontariens (jeunes de 18-25 ans) de la région d'Ottawa (Ontario, Canada) et basé sur le manuel de traitement SS (Najavits, 2002a). Ces analyses sont tirées d'une évaluation de programme qui cherchait à améliorer la qualité des services offerts dans le cadre de la Clinique Trauma et Psychiatrie Transculturelle (CTPT) de I'Université d'Ottawa - Programme de santé mentale de l'Hôpital Montfort (Clinique maintenant fermée pour des raisons administratives).

Les services étaient offerts aux adultes (18-65 ans) dans un format de groupe portant sur les 25 thèmes du programme SS. Ils étaient offerts une fois par semaine (durée de 3 heures), et ce, pour une période de six mois. II s'agissait donc d'un traitement assez intensif où le but principal était d'atteindre la sécurité et la stabilité dans les relations, les pensées, les comportements et les émotions. II est à noter que les services offerts ne s'adressaient pas exclusivement aux jeunes de 18-25 ans et que ces derniers côtoyaient des adultes. Des services individuels étaient aussi offerts à la CTPT; dont l'application du programme SS en format individuel. 
Entre autres choses, l'évaluation de programme cherchait à savoir si les services offerts dans le cadre de la CTPT semblaient efficaces pour les jeunes franco-ontariens. Les hypothèses de recherche étaient à l'effet que l'application du programme SS devrait permettre : (a) une diminution de la symptomatologie traumatique, (b) une diminution du taux de consommation de substances et, (c) une augmentation du niveau de fonctionnement général.

\section{Méthode}

Pour répondre aux objectifs de la présente évaluation de programme, un devis de recherche préexpérimentale de type prétest/post-test à groupe unique (Fortin \& Gagnon, 2010) a été appliqué. Ce type de devis présente l'avantage d'être facilement implantable en milieu clinique où les services à la clientèle ont préséance sur la rigueur méthodologique. Par ailleurs, et dans le cadre d'une première phase d'investigation scientifique dans le monde de la francophonie, ce type de devis permet une évaluation des effets bruts du programme. Évidemment, un tel devis comporte toutefois le désavantage d'être non discriminant sur le plan causal.

\section{Participants}

Les participants ( $N=9$ ) âgés de 18 à 25 ans étaient issus d'un bassin d'individus franco-ontariens $(N=47)$ de la région d'Ottawa qui ont bénéficié du programme $S S$ en groupe $(n=6)$ ou en séance individuelle $(n=3)$. La sélection de ces participants était tout simplement en lien avec ceux qui ont accepté l'application du programme SS à la suite d'une recommandation du psychiatre traitant, et ce, durant les années 2012 et 2013 et qui étaient âgés de moins de 25 ans. II est à noter que durant cette période, un jeune supplémentaire a retiré son consentement après l'avoir préalablement donné (sans participation au programme SS) et que deux autres jeunes ont abandonné le programme pour des raisons indépendantes de leur volonté (entrée en centre fermé de toxicomanie et déménagement). Les participants ont tous été diagnostiqués avec un ÉSPT et un TUS (actuel ou en rémission récente).

Sommairement, le groupe de neuf participants disponibles pour analyses dans le cadre de cette étude étaient composés de six femmes et de trois hommes âgés entre 18 et 25 ans $(\underline{M}=21,0$; $\underline{\underline{E} T}=2,70)$. Le principal statut social des participants avait trait au fait d'être étudiant(e) $(44,4 \%)$, de travailler (44,4\%) et d'être bénéficiaire d'une pension d'invalidité (11,1\%). En termes de revenu, la majorité des participants (77,8\%) gagnaient moins de $10000 \$$ par année, alors que 22,2\% gagnaient entre 10000 \$ et 20000 \$ par année. En ce qui concerne leur statut civil, $77,8 \%$ des participants étaient célibataires et $22,2 \%$ étaient en union libre. Ayant trait au type de logement, $55,6 \%$ demeuraient à la résidence de leurs parents, 33,3\% habitaient dans un appartement et $11,1 \%$ demeuraient dans un centre de traitement de la toxicomanie. Le diplôme académique complété le plus élevé par les participants était la $12^{\mathrm{e}}$ année $/ 5^{\mathrm{e}}$ secondaire $(44,4 \%)$, le primaire $(33,3 \%)$ et le collégial $(22,2 \%)$.

En matière de traumatismes vécus, les participants ont été confrontés à des agressions physiques $(33,3 \%)$, des agressions sexuelles $(44,4 \%)$ ou une combinaison agressions physiques et sexuelles (22,2\%); la plupart des agressions étant survenues dans l'enfance. Un participant a aussi été témoin d'évènements traumatiques à maintes reprises dans un contexte de vie familiale difficile. 


\section{Matériel}

Le matériel utilisé dans le cadre de la présente étude était en lien la version francophone du manuel de traitement SS (Najavits, 2002a). Le programme couvrait les 25 thèmes du manuel de traitement (ces derniers ont été directement travaillés en séance avec les intervenants et non simplement lus de façon indépendante par les participants). Le degré de conformité à l'égard de chaque séance a été évalué sur une échelle en trois points (non conforme, en partie conforme ou conforme), et ce, pour chacun des éléments suivants : (a) vérification initiale et gestion des enjeux abordés, (b) citation du jour et discussion sur le thème central, (c) distribution du matériel et utilisation en séance, (d) vérification finale avec engagement concret, (e) centration sur trauma/ÉSPT, (f) centration sur consommation de substances, (g) centration sur habilités saines et sécuritaires, (h) centration sur le thème du jour et pratique d'habiletés et (i) capacité à garder les participants en sécurité.

\section{Mesures}

Quelques tests psychologiques ont été sélectionnés et administrés en condition de pré et de posttraitement. II est à noter que tous les tests ont été administrés en français via des traductions publiées ou une traduction maison obtenue à l'aide d'une méthode de traduction de type comité (Brislin, Lonner \& Thorndike, 1973). Ces mesures présentent de bonnes propriétés psychométriques généralement reconnues dans le domaine.

Le Posttraumatic Stress Disorder Checklist Scale (PCL-S; Weathers et al., 1993) a été utilisé pour l'évaluation de l'ÉSPT alors que le Michigan Alcohol Screening Scale (MAST-22; Selzer, 1971) et le Drug Abuse Screening Test (DAST-20; Skinner, 1982) ont été utilisés pour l'évaluation du TUS. Pour l'évaluation du fonctionnement, le jugement clinique en ce qui a trait à l'évaluation globale du fonctionnement (EGF - Axe 5 du DSM-IV-TR, APA, 2000) a été utilisé.

\section{Procédure}

À la suite de la référence du psychiatre traitant (I.B.) et après acceptation de participer au programme de thérapie, les participants ont été invité(e)s à compléter quelques tests en prétest, et ce, dans une perspective d'évaluation de programme. Puis, ils ont été invités (e)s à participer à une ou deux rencontres d'entretien motivationnel selon une démarche standardisée et développée par un des auteurs du présent article et membre du MINT (Daoust, 2011). Par la suite et en fonction de la disponibilité d'accès au groupe (généralement rapide), les participants ont été invités à participer au groupe de thérapie en raison d'une séance de trois heures par semaine pour un total possible de 75 heures. Les séances de thérapie étaient coanimées par un psychologue et des étudiantes en formation (doctorat clinique en psychologie). Tous les participants, y compris les thérapeutes, s'assoyaient en rond dans une salle de thérapie et les jeunes participants côtoyaient d'autres participants adultes âgés de moins de 65 ans.

Toutes les séances se sont déroulées de la même manière et en conformité avec le manuel de traitement (Najavits, 2002a). Essentiellement, les séances se déroulaient selon quatre étapes. La première étape, la vérification initiale, permettait aux thérapeutes de vérifier comment les participants se sentaient au moment de la rencontre. Cinq minutes étaient accordées à chacundes participants et ces derniers répondaient à quelques questions. La deuxième étape était, quant à elle, en lien avec une citation du jour présélectionnée pour chacune des séances. Un participant devait alors lire celle-ci à haute voix et devait discuter du sens qu'elle avait pour lui. Le tout avait comme principal objectif d'engager émotionnellement les participants, d'introduire le thème du jour 
et pouvait aussi servir à titre d'élément d'inspiration future. La troisième étape, la plus importante en termes de durée et de contenu, portait sur un thème particulier relié à l'ÉSPT et au TUS. Les participants se voyaient alors distribué une copie du document propre à la séance et le contenu était travaillé en groupe via des lectures individuelles, des lectures à haute voix pour le groupe, des échanges, une mise en relation avec le vécu personnel et la pratique de jeux de rôles lorsque cela se révélait pertinent ou nécessaire. Finalement, la dernière étape en lien avec la vérification finale permettait de souligner et d'encourager les progrès accomplis par les participants et de fournir une rétroaction aux psychothérapeutes. Les participants devaient, là aussi, répondre à quelques questions et prendre un engagement envers eux-mêmes. À la fin du suivi, les participants ont complété la courte batterie de tests composant le post-test.

Le suivi individuel se déroulait de la même façon (animation par le psychologue de l'équipe) à l'exception de la durée des séances qui était réduite à soixante minutes (dose thérapeutique moins forte en terme de durée, mais plus forte en terme de concentration; $100 \%$ du temps étant accordé aux participants).

En ce qui concerne les analyses statistiques, des analyses descriptives et comparatives (test $t$ ) ont été effectuées. Étant donné le côté préliminaire de la présente étude, un niveau d'erreur de type I $(p \leq 0,05)$ relativement libéral a été considéré. En ce qui a trait à la grandeur d'effet des comparaisons effectuées, le calcul de la statistique $\underline{d}$ de Cohen (1988) a été retenu et les balises suivantes ont été élaborées pour guider son interprétation (autour de 0,2 = effet de petite taille; autour de 0,5 = effet de taille moyenne; autour de 0,8 et plus = effet de grande taille). La puissance statistique, quant à elle, a été calculée à partir de la formule développée par Faul, Erdfelder, Lang et Buchner (2007) et ce, pour une erreur de type 1 de $5 \%$.

\section{Résultats}

Avant de déterminer l'efficacité potentielle du programme offert, il importe de mentionner qu'il est ici assumé que le programme SS a été mis en œuvre de façon adéquate : bon degré de conformité avec le manuel de traitement (indice moyen de conformité estimé à 98,3\% dans l'étude de Daoust et al., 2014), bonne satisfaction auto-rapportée des participants, ajustement dyadique adéquat entre les psychothérapeutes dans la coanimation des séances et bonne disponibilité affective des psychothérapeutes (Daoust et al., 2014).

En termes de participation, les jeunes dans leur ensemble ont été présents en moyenne à $72,4 \%$ des séances. La durée moyenne des suivis s'est échelonnée sur 17,3 semaines (ÉT $=5,5$; Étendue : 12,0 à 24,0). En ce qui a trait aux jeunes impliqués dans un suivi de groupe seulement, ces derniers ont été présents aux séances dans une proportion de $75,3 \%$ et la durée moyenne des suivis s'est espacée sur 16,0 semaines (ÉT $=6,2$; Étendue : 12,0 à 24,0). Concernant les jeunes impliqués dans un suivi individuel, ces derniers ont été présents aux séances dans une proportion de $66,7 \%$ et la durée moyenne des suivis s'est espacée sur 20,0 semaines (ÉT $=2,6$; Étendue : 17,0 à 22,0).

En fonction du PCL-S et au moment du prétest, les participants présentaient tous une détresse traumatique significative ( $\underline{M}=64,1 ; \underline{\underline{T} T}=11,1$; Étendue : 47 à 80$)$. En ce qui a trait au TUS en début de suivi, les participants présentaient des difficultés en lien avec l'alcool $\underline{\underline{M}}=5,3$; $\underline{\underline{T}}=3,7$; Étendue : 0 à 12) au MAST-22 où 66,7\% d'entre eux étaient considérés comme des buveurs problématiques (problem drinker) et avaient des difficultés en lien avec l'utilisation de drogues ( $\underline{M}=8,6$; $\underline{T}=5,9$; Étendue : 0 à 16) au DAST-20 où $66,7 \%$ d'entre eux se définissaient comme ayant une sévérité 
de consommation intermédiaire, substantielle ou sévère. Ils présentaient tous des difficultés avec l'utilisation problématique de substances, et ce, à des degrés divers. Concernant le niveau de fonctionnement des participants en fonction de l'axe 5 du DSM-IV-TR (APA, 2000), les participants présentaient des symptômes importants $(\underline{M}=48,3$; $\underline{\underline{T}}=6,6$; Étendue : 40 à 60$)$ ayant un impact significatif sur leur fonctionnement et ce, au moment de leur admission dans la clinique. II est à noter que les évaluations du fonctionnement ont été réalisées par des cliniciens d'expérience (psychiatre ou psychologue).

Les tableaux 1 et 2 font état de l'évolution des variables dépendantes en fonction d'une participation à des séances de groupe ou individuelles. Les résultats suggèrent des améliorations significatives au PCL-S, au MAST-22 et en fonction de l'axe 5 (grandes tailles d'effet). Aucune amélioration statistiquement significative n'a cependant été notée pour la prestation de soins individuels et pour le DAST-20 en contexte de groupe.

Tableau 1 : Analyses comparatives entre le pré-test et le post-test pour l'ensemble des variables dépendantes de l'étude (intervention de groupe; $N=6$ ).

\begin{tabular}{|c|c|c|c|c|c|}
\hline Mesure & Moyenne pré & Moyenne post & Test $t$ & $\begin{array}{l}\text { Taille d'effet } \\
\text { (d de Cohen) }\end{array}$ & $\begin{array}{l}\text { Puissance } \\
\text { statistique }\end{array}$ \\
\hline \multicolumn{6}{|l|}{ Symptomatologie traumatique } \\
\hline PCL-S : Score total & 61,17 & 43,17 & 3,$212 ;$ dl $5 ; p<0,024^{*}$ & 1,33 & $90,3 \%$ \\
\hline PCL-S : Nombre critères " $B$ " & 3,83 & 3,17 & 1,$348 ;$ dl $5 ; p<0,235$ & 0,67 & $37,6 \%$ \\
\hline PCL-S : Nombre critères " $C_{\text {" }}$ & 4,17 & 3,50 & 1,$195 ;$ dl $5 ; p<0,286$ & 0,52 & $24,9 \%$ \\
\hline PCL-S : Nombre critères " $D$ " & 3,50 & 2,50 & 1,$732 ;$ dl $5 ; p<0,144$ & 0,71 & $41,7 \%$ \\
\hline \multicolumn{6}{|l|}{ Consommation de substance } \\
\hline MAST-22 : Alcool & 6,50 & 4,83 & 2,$988 ;$ dl $5 ; p<0,031^{*}$ & 1,24 & $85,7 \%$ \\
\hline DAST-20 : Drogues & 7,67 & 5,00 & 1,$581 ;$ dl $5 ; p<0,175$ & 0,75 & $44,8 \%$ \\
\hline \multicolumn{6}{|l|}{ Fonctionnement général } \\
\hline Axe 5 du DSM-IV-TR & 46,67 & 55,00 & $-2,712 ;$ dl $5 ; p<0,042^{*}$ & 1,11 & $77,3 \%$ \\
\hline
\end{tabular}

Note. * Amélioration significative pour un $\mathrm{p} \leq 0,05$.

Tableau 2 : Analyses comparatives entre le pré-test et le post-test pour l'ensemble des variables dépendantes de l'étude (intervention individuelle; $N=3$ ).

\begin{tabular}{|c|c|c|c|c|c|}
\hline Mesure & Moyenne pré & Moyenne post & Test $t$ & $\begin{array}{l}\text { Taille d'effet } \\
\text { (d de Cohen) }\end{array}$ & $\begin{array}{l}\text { Puissance } \\
\text { statistique }\end{array}$ \\
\hline \multicolumn{6}{|l|}{ Symptomatologie traumatique } \\
\hline PCL-S : Score total & 70,00 & 43,00 & 1,$764 ;$ dl $2 ; p<0,220$ & 1,01 & $41,4 \%$ \\
\hline PCL-S : Nombre critères "B" & 3,33 & 2,00 & 1,$512 ;$ dl $2 ; p<0,270$ & 1,03 & $43,1 \%$ \\
\hline PCL-S : Nombre critères “C” & 4,67 & 2,00 & 2,$000 ;$ dl $2 ; p<0,184$ & 1,16 & $51,9 \%$ \\
\hline PCL-S : Nombre critères “D” & 4,33 & 2,67 & 1,$387 ;$ dl $2 ; p<0,300$ & 0,99 & $40,1 \%$ \\
\hline \multicolumn{6}{|l|}{ Consommation de substance } \\
\hline MAST-22 : Alcool & 3,00 & 1,33 & 1,$387 ; \mathrm{dl} 2 ; p<0,300$ & 1,42 & $69,1 \%$ \\
\hline DAST-20 : Drogues & 10,33 & 5,33 & 1,$555 ;$ dl $2 ; p<0,260$ & 1,01 & $41,6 \%$ \\
\hline \multicolumn{6}{|l|}{ Fonctionnement général } \\
\hline Axe 5 du DSM-IV-TR & 51,67 & 61,67 & $-2,000 ; d l 2 ; p<0,184$ & 1,16 & $52,1 \%$ \\
\hline
\end{tabular}

Note. * Amélioration significative pour un $\mathrm{p} \leq 0,05$. 
Trauma et usage problématique de substances chez les jeunes

\section{Discussion}

Évidemment, cette étude est préliminaire et très limitée de par son petit nombre de participants. Par ailleurs, le devis utilisé est préexpérimental et ne comporte pas de groupe contrôle. Dans ce contexte, il importe d'être le plus critique et objectif possible. En fait, la plupart des analyses menées ne comportaient pas la puissance statistique nécessaire pour démontrer la présence d'un résultat statistiquement significatif si le traitement était en fait réellement efficace. Ainsi, le degré d'efficacité du programme SS pour les jeunes francophones ne peut être ici qu'au mieux estimé et aucun lien de causalité ne peut être tiré. Le fait que le nombre de résultats positifs (3) obtenus lors des analyses de comparaison prétest et post-test dépasse le nombre qui serait attendu uniquement par la chance ( $5 \%$ de 14 analyses, c.-à-d. : 0,7$)$ est en soi une condition nécessaire pour l'interprétation des présents résultats, mais non suffisante. Des études complémentaires devront donc être conduites avec plus de participants et un groupe contrôle, et ce, afin de pouvoir se prononcer avec plus de certitude sur l'efficacité du programme SS pour les jeunes francophones.

D'ici là, les résultats de la présente étude peuvent servir de fondement dans l'investigation scientifique de la pertinence du programme SS pour les jeunes et en particulier pour les francophones. II apparait premièrement que les participants ont été présents aux séances dans une proportion élevée (globale : 72,4\%; en groupe : $75,3 \%$ et en individuel : $66,7 \%$ ) et supérieure à une dose considérée comme thérapeutique (taux d'exposition à $24,0 \%$ des séances ou plus) dans la littérature dans le domaine (Najavits, 2002a). Ce haut taux de participation pourrait indirectement témoigner de l'intérêt des jeunes à l'égard du programme SS. II aurait d'ailleurs été intéressant de pouvoir ici évaluer l'alliance thérapeutique; élément potentiellement médiateur du degré de participation et de l'efficacité potentielle du traitement.

Les résultats de groupe semblent mettre en évidence une diminution statistiquement et cliniquement significative de la symptomatologie traumatique globale. En fait et en fonction des critères développés par Blanchard, Jones Alexander, Buckley \& Forneris (1996) et Monson, Gradus, Young-Xu, Schnurr, Price \& Schum (2008), les participants semblent avoir évolué d'une population dite clinique à une population dite normale (seuil critique de 44 au PCL-S) et avoir fait preuve d'un changement d'une étendue doublement cliniquement significatif (une augmentation ou une diminution de 10 points est généralement associée à une amélioration ou une exacerbation clinique significative). Bien que non statistiquement significative, l'évolution de la symptomatologie traumatique en fonction des critères B, $C$ et D de l'ÉSPT tel que conceptualisé par le DSM-IV-TR (APA, 2000) semble indiquer une certaine tendance à l'amélioration (tailles d'effet moyennes).

Toujours en fonction d'une analyse de groupe, il apparaît que la consommation d'alcool des participants semble s'être statistiquement et cliniquement améliorée en comparaison avec le point de départ; consommation évoluant du statut de buveur problématique au statut de buveur potentiellement problématique (rétablissement partiel). II est intéressant de noter ici qu'aucun(e) des participants ne s'est présenté en état d'ébriété au moment des séances. L'évolution de la consommation de drogues, quant à elle, s'est révélée non statistiquement significative bien qu'allant dans le sens d'une certaine tendance à l'amélioration (taille d'effet moyenne; passage d'une sévérité intermédiaire à une faible sévérité).

Finalement, il est intéressant de noter que les jeunes semblent s'être statistiquement et significativement améliorés dans leur fonctionnement général (l'Axe 5 passant d'une symptomatologie importante à une symptomatologie moyenne; rétablissement partiel). 
Pour le moment, il semble que la version française du programme SS est prometteuse dans le traitement de groupe ÉSPT-TUS pour une population clinique de jeunes canadien-français. Rappelons toutefois que des études complémentaires sont nécessaires afin de confirmer en bonne et due forme son efficacité et mieux comprendre les enjeux inhérents à son application.

À titre d'exemple, il serait bien que la question du dosage thérapeutique soit examinée. II est à noter que la durée du suivi de groupe dans le cadre de la présente étude ( 3 heures par semaine = suivi intensif) a davantage été influencée par des craintes administratives que cliniques. Ceci dit, il est possible que le triple de la dose thérapeutique habituelle ( 3 heures vs 50 minutes) puisse avoir eu en soi un effet sur le cheminement des participants; une question que la présente étude n'est pas en mesure de répondre. II faudrait aussi que des études complémentaires viennent départager la contribution relative de l'entretien motivationnel et de la pharmacothérapie. II semble également pertinent de se demander si le mixage entre les jeunes adultes et les adultes plus âgés (26 ans et plus) est bénéfique étant donné les possibilités de modelage ou si les jeunes auraient pu encore plus tirer avantage d'un groupe leur étant uniquement destiné.

En ce qui a trait aux résultats en lien avec une prestation de services individuels, ils sont évidemment aussi très préliminaires $(n=3)$ et relèvent pratiquement du registre de l'étude de cas. Aucun résultat statistiquement significatif n'a pu être noté; ce qui pourrait laisser croire à une non-efficacité du programme SS pour les jeunes francophones en format individuel. Ceci dit, il est possible que les résultats de la présente étude ne rendent pas tout à fait justice à la réalité clinique des personnes concernées. En fait et de façon anecdotique, les trois participants se disaient très satisfaits des services offerts et rapportaient en avoir tiré bénéfice; impression aussi partagée par le thérapeute. Par ailleurs, il semble que toutes les mesures récoltées soient allées dans le sens d'une certaine tendance à l'amélioration où de grandes tailles d'effet pourraient caractériser le changement potentiel. II est donc possible ici qu'une faible puissance statistique empêche de mettre en évidence ce qui pourrait être considéré comme des changements significatifs et positifs. Des études complémentaires plus poussées avec un plus grand nombre de participants seront évidemment nécessaires pour clarifier la situation.

\section{Conclusion}

En conclusion, la mise en place du programme SS pour ÉSPT-TUS semble possible pour les jeunes francophones et pourrait induire des changements positifs, et ce, en lien avec des dimensions clés telles que la symptomatologie traumatique, la consommation d'alcool et le fonctionnement général. Ceci dit, la présente étude comportait des limites importantes. À cet égard, il est possible d'envisager que des variables extérieures, qui n'étaient pas sous le contrôle des évaluateurs, auraient pu avoir une influence sur l'amélioration des participants. À ce propos, il faut mentionner que les participants étaient sous les soins d'un psychiatre et que les facteurs pharmacologiques n'ont pas été pris en considération dans le cadre de la présente étude. À cela, il faut aussi ajouter un certain facteur confondant en lien avec la tenue de rencontres d'entretien motivationnel au préalable à la participation au programme SS.

Quant à l'évaluation de l'impact du programme SS à plus long terme, les résultats de la présente étude ne permettent pas de se prononcer à ce niveau, ce qui constitue une limite supplémentaire.

Tout compte fait, la contribution de l'étude est petite, mais relativement intéressante en ce qui a trait au traitement intégré du trouble concomitant ÉSPT-TUS pour une population clinique de jeunes francophones, et ce, puisqu'il semble s'agir, à notre connaissance du moins, des premiers 
résultats à être publiés dans la francophonie. Puisque la présente étude semble mettre en évidence des résultats potentiellement positifs, il est à penser que de futures études utilisant un plan de recherche plus complexe sont justifiées et à prévoir. Par ailleurs et d'un point de vue clinique, il pourrait être bien de considérer l'implantation d'une phase 2 dans le traitement ÉSPT-TUS (Creating Change; Najavits, en préparation) chez les jeunes et d'en évaluer, là aussi, l'efficacité. 


\section{Références}

American Psychiatric Association. (2000). Diagnostic and statistical manual of mental disorders ( $4^{\text {th }}$ ed., text revision). Washington, DC: Auteur.

American Psychiatric Association. (2013). Diagnostic and statistical manual of mental disorders ( $5^{\text {th }}$ ed.). Washington, DC: Auteur.

Blanchard, E.B., Jones Alexander, J., Buckley, T.C., \& Forneris, C.A. (1996). Psychometric properties of the PTSD Checklist (PCL). Behaviour Research and Therapy, 34, 669-673.

Boden, M.T., Kimerling, R., Jacobs-Lentz, J., Bowman, D., Weaver, C., Carney, D., Walser, R., \& Trafton, J. A. (2011). Seeking Safety treatment for male veterans with a substance use disorder and PTSD symptomatology. Addiction, 107(3), 578-586.

Brady, K.T., Dansky, B.S., Back, S.E., Foa, E.B., \& Carroll, K.M. (2001). Exposure therapy in the treatment of PTSD among cocaine-dependent individuals: Preliminary findings. Journal of Substance Abuse treatment, 21, 47-54.

Brady, K.T., Killeen, T., Saladin, M.E., Dansky, B., \& Becker, S. (1994). Comorbid substance abuse and posttraumatic stress disorder: Characteristics of women in treatment. American Journal on Addictions, 3, 160-164.

Breslau, N., Davis, G.C., Andreski, P., Peterson, E.L, \& Schultz, L.R. (1997). Sex Differences in Posttraumatic Stress Disorder. Archives of General Psychiatry, 54(11), 1044-1048.

Briere, J. (1995). Trauma Symptom Inventory professional manual. Odessa, FL: Psychological Assessment Resources.

Briere, J.N. (2000). Cognitive Distortion Scales: Professionnal Manual, Psychological Assessment Resources, Inc., 1-35.

Briere, J., \& Zaidi, L. (1989). Sexual abuse histories and sequelae in female psychiatric emergency room patients. American Journal of Psychiatry, 146, 1602-1606.

Brislin, R., Lonner, W., \& Thordike, R. (1973). Cross-cultural research methods. New-York: John Wiley.

Brown, P.J., Stout, R.L., \& Gannon-Rowley, J. (1998). Substance use disorder - PTSD co-morbidity: Patients' perception of symptom interplay and treatment issues. Journal of Substance Abuse Treatment, 15, 445-448.

Cohen, J.A., Mannarino, A.P, Zhitov, A.C., \& Capone, M.E. (2003). Treating child abuse-related posttraumatic stress and comorbid substance abuse in adolescents. Child Abuse \& Neglect, 27, 1345-1365.

Cohen, J. (1988). Statistical Power analysis for the behavioral science $\left(2^{\text {nd }}\right.$ ed.). Hillsdale, NJ: Lawrence Earlbaum Associates.

Cohen, L.R., \& Hien, D.A. (2006). Treatment outcomes for women with substance abuse and PTSD who have experienced complex trauma. Psychiatric Services, 57(1), 100-106. 
Cohen, Y., Spirito, A., \& Sterling, C. (1996). Physical and sexual abuse and their relation to psychiatric disorder and suicidal behaviour among adolescents who are psychiatrically hospitalized. Journal of Child Psychology and Psychiatry, 37, 989-993.

Cook, J.M., Walser, R.D., Kane, V., Ruzek, J.I., \& Woody, G. (2006). Dissemination and feasibility of a cognitive-behavioral treatment for substance use disorders and posttraumatic stress disorder in the Veterans Administration. Journal of Psychoactive Drugs, 38, 89-92.

Cottler L.B., Compton W.M., $3^{\text {rd }}$, Mager D., Spitznagel, E.L., Janca, A. (1992) Posttraumatic stress disorder among substance users from the general population. American Journal of Psychiatry, 149, 664-670.

Dansky, B., Saladin, M., Brady, K., Kilpatrick, D., \& Resnick, H. (1995). Prevalence of victimization and posttraumatic stress disorder among women with substance use disorders: A comparison of telephone and in-person assessment samples. The International Journal of the Addictions, 30, 1079-1099.

Daoust, J.-P. (2011). Séquence d'entretien motivationnel - Protocole d'intervention à double étapes version 2.1. Document inédit.

Daoust, J.-P., Renaud, M., Bruyère, B., Juery, C., \& Najavits, L. (2015). État de stress post-traumatique et usage problématique de substance : Évaluation de programme préliminaire en lien avec l'efficacité d'une clinique spécialisée pour Franco-ontariens en milieu hospitalier universitaire. Revue Psy Cause, 65.

Deblinger, E., \& Heflin, A.H. (1996). Treatment for sexually abused children and their nonoffending parents: A cognitive-behavioral approach. Thousand Oaks, CA: Sage Publications.

Deblinger, E., Lippman, J., \& Steer, R.A. (1996). Treatment for sexually abused children suffering post-traumatic stress symptoms: Initial treatment outcome finding. Child Maltreatment, 1, 310-321.

Desai, R.A., Harpaz-Rotem, I., Najavits, L.M., \& Rosenheck, R.A. (2009). Seeking Safety therapy: Clarification of results. Psychiatric Services, 60(1), 125.

Desai, R.A., Harpaz-Rotem, I., Najavits, L.M., \& Rosenheck, R.A. (2008). Treatment for homeless female veterans with psychiatric and substance abuse disorders: Impact of "Seeking Safety" on one-year clinical outcomes. Psychiatric Services, 59(9), 996-1003.

Donovan, B., Padin-Rivera, E., \& Kowaliw, S. (2001). Transcent : initial outcomes from posttraumatic stress disorder substance abus treatment program. Journal of Traumatic Stress, 14(4), 757-772.

Faul, F., Erdfelder, E., Lang, A.-G., \& Buchner, A. (2007). G*Power 3: A flexible statistical power analysis program for the social, behavioral, and biomedical sciences. Behavior Research Methods, 39, 175-191.

Fortin, M.-F., \& Gagnon, J. (2010). Fondements et étapes du processus de recherche - Méthodes quantitatives et qualitatives ( $2^{\text {nd }} \mathrm{ed}$.). Montréal : Chenelière Éducation.

Fullilove, M.T., Fullilove, R.E., Smith, M., Winkler, K., Michael, C., Panzer, P.G., \& Wallace, R. (1993). Violence, trauma, and post-traumatic stress disorder among women drug users. Journal of Traumatic Stress, 6, 533-543. 
Giaconia, R.M., Reinherz, H.Z., Hauf, A.C., Paradis, A.D., Wasserman, M.S., \& Langhammer, D.M., (2000). Comorbidity of Substance Use and Post-Traumatic Stress Disorders in a Community Sample of Adolescents. American Journal of Orthopsychiatry, 70(2), 253-262.

Giaconia, R.M., Reinherz, H.Z., Paradis, A.D., \& et al. (2002). Comorbidity of Substance Use and Post-Traumatic Stress Disorders in Adolescents. Dans P. Ouimette \& P. J. Brown (Eds.), Trauma and substance abuse: Causes, consequences and treatment of co-morbid disorders (pp. 209-226). Washington, D.C.: American Psychological Association.

Hamilton, N. (2006). African-Americam Center for Excellence (AACE) Program; SAMHSA grant number TI14126; final report. Operation PAR, Largo: FL. Rapport non publié,

Herman, J.L., Russell, D.E.H, \& Trocki, K. (1986). Long-term effects of incestuous abuse in childhood. American Journal of Psychiatry, 143, 1293-1296.

Hien, D. (2009). Trauma Services for Women in Substance Abuse Treatment : an intergrated Approach. Washington, D. C.: American Psychological Association.

Hien, D., \& Scheier, J. (1996). Trauma and short-term outcome for women in detoxification. Journal of Substance Abuse Treatment, 13(3), 227-231. Journal of the Addictions, 30, 1079-1099.

Hien, D.A., Cohen, L.R., Litt, L.C., Miele, G.M., \& Capstick, C. (2004). Promising empirically supported treatments for women with comorbid PTSD and substance use disorders. American Journal of Psychiatry, 161(8), 1426-1432.

Kessler, R.C., Sonnega, A., Bromet, E., Hughes, M. \& Nelson, C.B. (1995). Posttraumatic stress disorder in the National Comorbidity Survey. Archives of General Psychiatry. 52, 1048-1060.

Kilpatrick, D.G., \& Saunders, B.E. (1997). Prevelance and Consequences of Child Victimization: Results from the National Survey of Adolescents - Final Report. U.S. Department of justice.

Kulka, R.A., Schlenger, W.E., Fairbank, J.A., Hough, R.L., Jordan, B.K., Marmar, C.R., et al. (1990). Trauma and the Vietnam War generation: Report of findings from the National Vietnam Veterans Readjustment Study. New York: Brunner/Mazel.

Liddle, H.A., \& Hogue, A. (2001). Multidimensional family therapy for adolescent substance abuse. Dans Wayne, E.F., \& Waldron, H.B. (Eds), Innovations in adolescent substance abuse interventions (pp. 229-261). Oxford: Pregamon.

Lynch, S.M., Heath, N.M., Matthews, K.C., \& Cepeda, G.J. (2012). Seeking Safety: An Intervention for Trauma-Exposed Incarcerated Women? Journal of Trauma \& Dissociation, 13(1), 88-101.

Miller, B.A., Downs, W.R., Gondoli, D.M., \& Keil, A. (1987). The role of childhood sexual abuse in the development of alcoholism in women. Violence and Victims, 2, 157-172.

Miller, B.A., Downs, W.R., \& Testa, M. (1993). Interrelationships between victimization experiences and women's alcohol use. Journal of Studies on Alcohol, supplement no. 11, 109-117.

Monson, C.M., Gradus, J.L., Young-Xu, Y., Schnurr, P.P, Price, J.L., \& Schumm, J.A. (2008). Change in posttraumatic stress disorder symptoms: Do clinicians and patients agree? Psychological Assessment, 20(2), 131-138. 
Najavits, L. (2003). Seeking Safety : a New Psychotherapy for Posttraumatic Stress Disorder and Substance Use Disorder. Dans P. Ouimette \& P. J. Brown (Eds.), Trauma and substance abuse : Causes, consequences and treatment of co-morbid disorders (pp. 147-169). Washington, D.C.: American Psychological Association.

Najavits, L.M. (2002a). Seeking Safety: A Treatment Manual for PTSD and Substance Abuse. New York, NY: The Guilford Press.

Najavits, L.M. (2002b). A Woman's Addiction Workbook. Oakland, CA : New Harbinger.

Najavits, L.M., Gallop, R.J., \& Weiss, R.D. (2006). Seeking Safety Therapy for Adolescent Girls with PTSD and Substance Use Disorder: A Randomized Controlled Trial. Journal of Behavioral Health Services \& Research, 33(4), 453-463.

Najavits, L.M., \& Hien, D. (2013). Helping Vulnerable Populations: A Comprehensive Review of the Treatment Outcome Literature on Substance Use Disorder and PTSD. Journal of clinical psychology, 69(5). 433-479.

Najavits, L.M., Schmitz, M., Gotthardt, S., \& Weiss, R.D. (2005). Seeking Safety plus Exposure Therapy for Dual Diagnosis Men. Journal of Psychoactive Drugs, 27, 425-435.

Najavits, L.M., Schmitz, M., Johnson, K.M., Smith, C., North, T., Hamilton, N., Walser, R., Reeder, K. Norman, S., \& Wilkins, K.. (2009). Seeking Safety therapy for men: Clinical and research experiences. Dans : Katlin, L.J., Men and Addictions. Nova Science Publishers, Hauppauge, NY.

Najavits, L.M., Weiss, R.D., Shaw, S.R., \& Muenz, L.R. (1998). "Seeking Safety ": Outcome of a New Cognitive-Behavioral Psychotherapy for Women with Posttraumatic Stress Disorder and Substance Dependence. Journal of Traumatic Stress, 11(3), 437 - 456.

Norman, S.B., Wilkins, K.C., Tapert, S.F., Lang, A.J., \& Najavits, L M. (2010). A pilot study of seeking safety therapy with OEF/OIF veterans. Journal of Psychoactive Drugs, 42, 83-87.

Ouimette, P., Brown, P.J. (2002). Trauma and Substance Abuse: Causes, Consequences, and Treatment fo Comorbid Disorders. Washington, D.C.: American Psychological Association.

Ouimette, P., Moos, R.H., \& Brown, P.J. (2003). Substance use disorder-posttraumatic stress disorder co-morbidity : A survey of treatments and proposed practice guidelines. Dans $P$. Ouimette $\& P$ J. Brown (Eds.), Trauma and substance abuse: Causes, consequences and treatment of co-morbid disorders (1 ${ }^{\text {st }}$ ed., pp. 91-111). Washington, D.C.: American Psychological Association.

Paglia-Boak, A., Adlaf, E.M., \& Mann, R.E. (2011). Drug use among Ontario students, 1977-2011: Detailed OSDUHS findings (CAMH Research Document Series No. 32). Toronto, ON: Centre for Addiction and Mental Health.

Paglia-Boak, A., Adlaf, E.M., Hamilton, H.A., Beitchman, J.H., Wolfe, D., \& Mann, R.E. (2012). The mental health and well-being of Ontario students, 1991-2011 : Detailed OSDUHS findings (CAMH Research Document Series No. 34). Toronto, ON: Centre for Addiction and Mental Health.

Reiger, D.A., Farmer, M.E. \& Rae, D.S. (1990). "Co-morbidity of mental disorders with alcohol and other drug abuse, Results from the Epidemiological Catchment Area (ECA) study ", Journal of the American Medical Association, 264, 2511-2518. 
Riggs, P.D., \& Davies, R.D. (2002). A clinical approach to integrating treatment for adolescents depression and substance abuse. Journal of the American Academy of Child and Adolescent Psychiatry, 41, 1253-1255.

Riggs, D.S., \& Foa, E.B. (2008). Treatment for Co-morbid Posttraumatic Stress Disorder and Substance Use Disorder. Dans S. H. Stewart \& P. J. Conrod (Eds.), Anxiety and Substance Use Disorder: The Vicious Cylce of Comorbidity (pp. 119-137). Boston, MA: Springer.

Selzer, M.L. (1971) 'The Michigan Alcoholism Screening Test (MAST): The quest for a new diagnostic instrument', American Journal of Psychiatry, 127, 1653-1658.

Skinner, H. A. (1982). The Drug Abuse Screening Test. Addictive Behaviors, 7, 363-371.

Skinner, W.J., O'Grady, C.P., Bartha, C., \& Parker, C. (2004). Les troubles concomitants de toxicomanie et de santé mentale : guide d'information. Canada : Centre de toxicomanie et de santé mentale.

Simoneau, H., \& Guay, S. (2008). Conséquences de la concomitance des troubles liés à l'utilisation de substances et à l'état de stress post-traumatique sur le traitement. Drogues, santé et société, $7(2), 125-160$.

Stewart, S.H., \& Conrod, P.J. (2008). Anxiety disorders and substance use disorders co-morbidity: common themes and future directions. Dans Stewart, S.H., \& Conrod, P.J. Anxiety and Substance Use Disorders: The Vicious Cycle of Comorbidity (pp. 239-257). New York: Springer.

Triffleman, E., Carroll, K., \& Kellogg, S. (1999). Substance dependence posttraumatic stress disorder therapy: An integrated cognitive-behavioral approach. Journal of Substance Abuse Treatment. 17, 3-14.

Weathers, F. W., Litz, B. T., Herman, D. S., Huska, J. A., \& Keane, T. M. (1993). The PTSD Checklist $(P C L)$ : Reliability, validity, and diagnostic utility. Paper presented at the 9th Annual Conference of the ISTSS, San Antonio, TX.

Weaver, C M., Trafton, J.A., Walser, R.D., \& Kimerling, R.E. (2007). Pilot test of Seeking Safety with male veterans. Psychiatric Services, 58, 1012. Also, see reply: Najavits, L. M. (2007). Letter to the editor: Reply to Weaver et al. (2007). Psychiatric Services, 58, 1376.

Weller, L.A. (2005). Group therapy to treat substance use and traumatic symptoms in female veterans. Federal Practitioner, 22(4), 27-38.

Zlotnick, C., Johnson, J., \& Najavits, L.M. (2009). Randomized controlled pilot study of cognitive behavioral therapy in a sample of incarcerated women with substance use disorder and PTSD. Behavior Therapy, 40(4), 325-36.

Zlotnick, C., Najavits, L.M., Rohsenow, D.J., \& Johnson, D.M. (2003). A cognitive-behavioral treatment for incarcerated women with substance use disorder and posttraumatic stress disorder: Findings from a pilot study. Journal of Substance Abuse Treatment, 25. 99-105 